\title{
Treatment of Children and Adolescents with Attention Deficit Hyperactivity Disorder and/or Tourette's Disorder with Clonidine Extended Release
}

\author{
Sung Woo Joo and Hyo-Won Kim ${ }^{\bowtie}$ \\ Department of Psychiatry, University of Ulsan College of Medicine, Asan Medical Center, Seoul, Republic of Korea
}

We aimed to assess the effectiveness and safety of clonidine extended release (ER) treatment in Korean youth with ADHD and/or Tourette's disorder. We retrospectively reviewed the medical records of 29 children and adolescents treated with clonidine ER. The effectiveness were retrospectively measured at baseline and after 4 and 12 weeks based on the Clinical Global Impression-Severity (CGI-S) and Clinical Global Impression-Improvement (CGI-I) scores. Safety was evaluated at each visit based on spontaneous reports from the subjects or from their parents/guardians. Significant decreases in the CGI-S scores for both ADHD (F=23.478, $\mathrm{p}<0.001, \mathrm{partial} \eta 2=0.540)$ and tic symptoms $(\mathrm{F}=15.137, \mathrm{p}<0.001$, partial $\eta 2=0.443)$ were noted over 12 weeks. The most common adverse event was somnolence $(n=9,31.0 \%)$ and life-threatening adverse effects were not observed. Our results provide preliminary evidence for the effectiveness and safety of clonidine ER.

Psychiatry Investig 2018;15(1):90-93

Key Words ADHD, Clonidine extended release, Tourette's disorder.

\section{INTRODUCTION}

The immediate release (IR) formulation of clonidine has been used off-label for many years to treat attention-deficit/hyperactivity disorder (ADHD). ${ }^{1}$ Recently, clonidine extended release (ER) was approved by the Korean and United States (US) Food and Drug Administration (FDA) for the treatment of $\mathrm{ADHD}$ in children and adolescents. A recent meta-analysis reported that clonidine, either alone (Hedges' $\mathrm{g}=-0.44)$ or in combination with stimulants (Hedges'g $=-0.32$ ), was significantly superior to placebo for ADHD symptoms. Although $\alpha-2$ agonists including clonidine, guanfacine have lesser efficacy than psychostimulants, $\alpha-2$ agonists reportedly have similar effect sizes of around 0.6 as atomoxetine. ${ }^{2,3}$ Although some subjects experience adverse effects including fatigue, somnolence/

\footnotetext{
Received: August 5, 2016 Revised: February 17, 2017

Accepted: April 17, 2017 Available online: November 16, 2017

$\triangle$ Correspondence: Hyo-Won Kim, MD, PhD

Department of Psychiatry, University of Ulsan College of Medicine, Asan Medical Center, 88 Olympic-ro 43-gil, Songpa-gu, Seoul 05505, Republic of Korea

Tel: +82-2-3010-3414, Fax: +82-2-485-8381,

E-mail: shingubi@amc.seoul.kr

(c) This is an Open Access article distributed under the terms of the Creative Commons Attribution Non-Commercial License (http://creativecommons.org/licenses/bync/4.0) which permits unrestricted non-commercial use, distribution, and reproduction in any medium, provided the original work is properly cited.
}

sedation, hypotension, bradycardia, and possibly QTc prolongation, treatment with clonidine ER is generally well tolerated. Clonidine is also considered as the first-line therapy for Tourette's syndrome in the United States and Canada. ${ }^{4}$ Several recent studies have shown that clonidine, either as an ER formulation or an adhesive patch, has beneficial effects both in youth and adults with tic disorders, particularly Tourette's disorder. ${ }^{5,6}$

However, to our knowledge, only a few previous studies have been performed in an Asian population. We aimed to investigate the effectiveness and safety of clonidine ER treatment in Korean children and adolescents.

\section{METHODS}

\section{Subjects}

This study was approved by the Institutional Review Board of Asan Medical Center (IRB No. S2015-0874). We retrospectively reviewed the medical records of 29 children and adolescents who were treated with clonidine ER from November 2013 to September 2015 at the Department of Psychiatry, Asan Medical Center, Seoul, Korea. For inclusion in our analyses, all subjects had to meet the following criteria: 1) aged between 5 and 18 years and 2) underwent at least 1 assessment for effec- 
tiveness and safety. The subjects were excluded based on the following criteria: 1) the presence of major neurological disorders; 2) poor compliance.

Two independent psychiatrists (H.W.K and S.W.J) retrospectively obtained the data from each subject. Diagnoses and comorbid psychiatric disorders were assessed according to the Diagnostic and Statistical Manual of Mental Disorders, 5th edition (DSM-5) (American Psychiatric Association 2013) criteria.

\section{Effectiveness and safety of clonidine ER}

To assess the effectiveness of clonidine ER, the Clinical Global Impression of Severity (CGI- S) and Clinical Global Impression of Improvement (CGI-I) scores were retrospectively determined by two independent psychiatrists (H.W.K and S.W.J). Information within the medical records included inattention, hyperactivity, impulsivity and tics as well as functional impairment caused by those symptoms such as peer relationship, academic functioning, relationship with teachers, and relationship with parents. The CGI-S values were recorded at baseline, and the CGI-S and CGI-I scores were also recorded after 4 and 12 weeks. The CGI-S and CGI-I scores for ADHD and tics were separately scored. Inter-rater correlation was evaluated by Cohen's kappa coefficient, which was 0.85 . Treatment response was defined as a CGI-I score of $\leq 2$ by the 12 th week of follow-up. Safety was evaluated at each visit based on spontaneous reports from the subjects or from their parents/guardians.

\section{Statistical analyses}

Statistical analyses were performed using PASW (version 21.0, SPSS Inc., Chicago, IL, USA), and all comparisons were two-tailed. A significance level of 0.05 was used. Demographic characteristics and psychiatric comorbidities were compared using the chi-squared or Fisher's exact tests (categorical variables), Student t-test (dimensional variables), and KruskalWallis test (ordinal variables). The repeated measures analysis of variance (ANOVA) was used to analyze the time effect (baseline and after 4 and 12 weeks). Missing data were imputed by the last observation carried forward (LOCF).

\section{RESULTS}

Twenty-nine subjects were included in our analysis, with a mean age of $11.1 \pm 3.0$ years (range, 5.2-17.8 years) and a predominance of males $(n=28,96.6 \%)$. The mean duration in the entire treatment period was $211.7 \pm 255.4$ days (range, 4-1,299 days). The mean dose in the 12 weeks treatment and the entire treatment period were $0.13 \pm 0.05$ (range, $0.1-0.29 \mathrm{mg} /$ day) and $0.14 \pm 0.06 \mathrm{mg} /$ day (range, $0.1-0.29 \mathrm{mg} /$ day), respectively. Demographic and clinical characteristics of subjects are provided in Supplementary Table 1 (in the online-only Data Supplement).

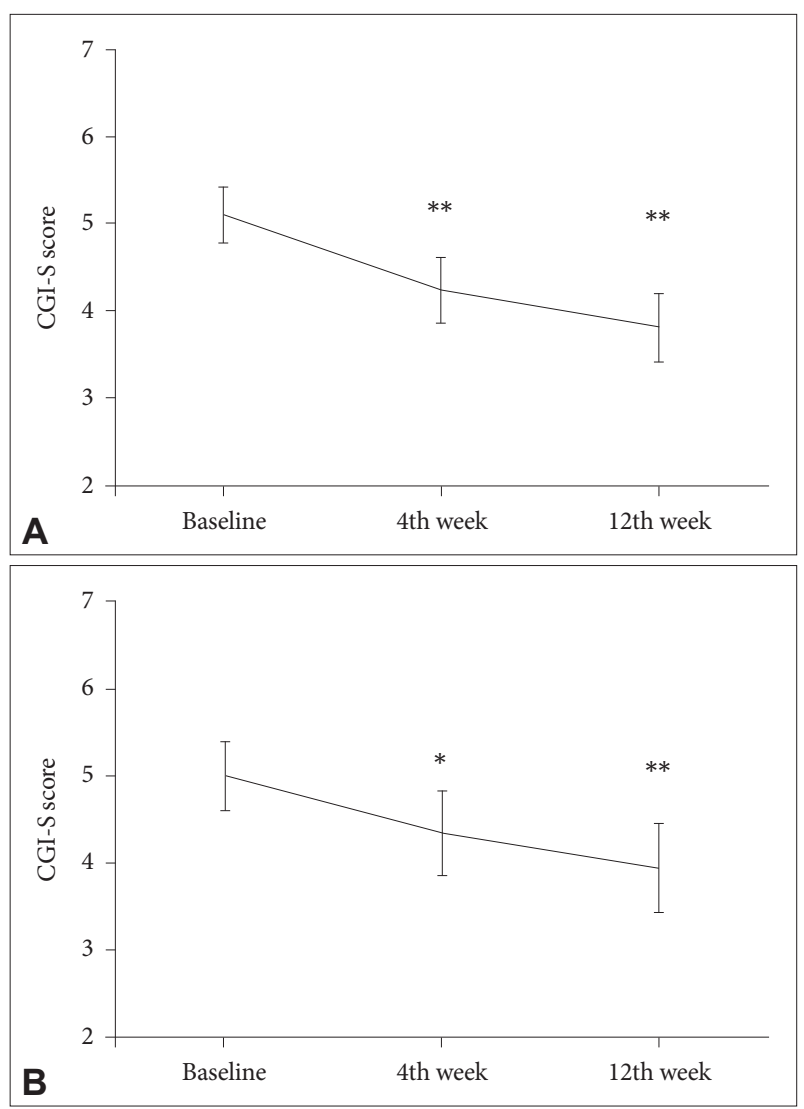

Figure 1. Mean values and confidence intervals of the Clinical Global Impressions-Severity (CGI-S) scores. (A) ADHD and (B) tic symptoms. ${ }^{*} p<0.05,{ }^{* *} p<0.001$. ADHD: attention-deficit/hyperactivity disorder.

Figure 1 illustrates the mean scores and standard errors obtained from a repeated measure ANOVA of the CGI-S scores for ADHD and tic symptoms at baseline, 4th week and 12th week. Of the 22 subjects who had ADHD, 17 had a prior history of insufficient response to psychostimulants and/or atomoxetine. The CGI-S scores for ADHD symptoms were significantly decreased during the 12-week clonidine ER treatment $(\mathrm{F}=23.478, \mathrm{p}<0.001$, partial $\eta 2=0.540)$, and $7(31.8 \%)$ of 22 subjects showed a treatment response. Of the 20 subjects who had tic disorder, 16 had an inadequate response to antipsychotics prior to clonidine ER. The CGI-S scores for tic symptoms were significantly improved during the 12-week clonidine $E R$ treatment $(\mathrm{F}=15.137, \mathrm{p}<0.001$, partial $\eta 2=0.443)$, and 5 (25.0\%) of 20 subjects exhibited a treatment response.

Nineteen (65.5\%) subjects reported adverse effects. The most common adverse effect was somnolence $(n=9,31.0 \%)$, followed by dizziness $(n=5,17.2 \%)$, fatigue $(n=1,3.4 \%)$, insomnia $(\mathrm{n}=1,3.4 \%)$, night terrors $(\mathrm{n}=1,3.4 \%)$, hypotension $(\mathrm{n}=1$, $3.4 \%)$, nausea $(n=1,3.4 \%)$, chest discomfort $(n=1,3.4 \%)$, headache $(\mathrm{n}=1,3.4 \%)$, and aggravation of motor and vocal tics ( $\mathrm{n}=$ $1,3.4 \%)$. The somnolence was tolerable in seven of the nine 
subjects, whereas the other two subjects discontinued clonidine ER. One subject experienced decreased systolic and diastolic blood pressure (BP) (83/45 mm Hg), during dose titration from $0.2 \mathrm{mg} /$ day to $0.3 \mathrm{mg} /$ day, which spontaneously resolved. Serious adverse events were not reported. Six (20.7\%) subjects discontinued the medication within 12 weeks due to adverse effects; dizziness in two cases, and somnolence, night terror, nausea, and aggravation of motor and vocal tics in one subject each. Additionally, two subjects discontinued the clonidine ER due to insufficient effectiveness.

\section{DISCUSSION}

In this retrospective chart review, we found that clonidine ER was effective and generally tolerable in children and adolescents with ADHD and/or Tourette's disorder. To our knowledge, this is the only study to evaluate the effectiveness of clonidine ER in Korean children and adolescents with ADHD and/ or Tourette's disorder.

Consistent with previous studies, our findings indicated that ADHD symptoms were significantly improved during the 12 weeks treatment period. A randomized, placebo-controlled study showed that clonidine ER is useful in reducing ADHD symptoms and is generally well tolerated. ${ }^{7}$ Moreover, in subjects with a partial response to stimulants, the use of clonidine ER as an add-on therapy is beneficial in that it further improves the ADHD symptoms. ${ }^{8}$ As clonidine ER could help reduce hyperactivity and impulsivity and could promote sleep in the evening, it has also been considered as a useful medication for individuals who cannot tolerate psychostimulants or exhibit an insufficient response to psychostimulants.

In youth with Tourette's disorder, clonidine IR is reportedly useful for reducing tic and other behavioral symptoms. ${ }^{9} \mathrm{Re}-$ cently, treatment with a clonidine adhesive patch was reported to be superior to haloperidol therapy, with a safer and better-tolerated profile. ${ }^{5}$ However, the evidence regarding the effectiveness of clonidine ER in the treatment of Tourette's disorder is scarce. In the present study, the response rate to clonidine ER was $25.0 \%$ in subjects with tics; in fact, most subjects had exhibited an insufficient response to antipsychotics prior to treatment with clonidine ER. Thus, our results suggest that clonidine ER could be beneficial for subjects with an inadequate response to antipsychotics. However, one of our subjects exhibited aggravation of motor and vocal tics. Consistent with this finding, another study showed that the administration of clonidine led to the exacerbation of tics with severe systemic heat sensations. ${ }^{10}$ The reason for such adverse effects cannot be explained, as clonidine has been commonly used as a ticsuppressing agent. The effect of clonidine is known to be mediated via binding to $\alpha-2 \mathrm{~A}, 2 \mathrm{~B}$, and $2 \mathrm{C}$ receptors and is asso- ciated with inhibitory learning due to reduced noradrenergic activity. ${ }^{1}$ Our findings suggest that clonidine may also have another mode of action. Hence, the effectiveness of clonidine in tic disorders and its mode of tic suppression need to be further elucidated.

In our study, 19 (65.5\%) subjects experienced adverse effects; the most common adverse effect was somnolence, which subsequently resolved in most cases. These findings are similar to those of previous studies, wherein the percentages of treatment-emergent adverse events ranged from $62 \%$ to $70 \%$ in ADHD subjects treated with clonidine ER monotherapy and where somnolence and fatigue were the prominent side effects reported. ${ }^{5}$ We observed that six subjects (20.7\%) discontinued the medication due to adverse effects; this early discontinuation rate was also noted in previous studies. ${ }^{7}$ In our study, one subject exhibited decreases in systolic and diastolic BP during the dose titration period. Minor reductions in BP and heart rate, which were not considered as clinically significant, were also observed in previous studies. ${ }^{7,8}$ As these cardiovascular effects of clonidine are known to be dose-dependent, ${ }^{7}$ slow titration to an optimal dosage is recommended.

There were several notable limitations to our analyses. First, this study was designed as a retrospective chart review. Second, we did not include a placebo group. Hence, it is possible that the present findings may be the result of a placebo effect rather than an intrinsic effect of clonidine ER. Third, although we tried to minimize the effect of concomitant medication by performing analyses without ten subjects whose dose of concomitant medication was changed, which showed the effect of clonidine ER treatment on $\mathrm{ADHD}(\mathrm{F}=15.586, \mathrm{p}<0.001$, partial $\eta 2=0.545)$ and tic symptoms $(\mathrm{F}=9.556, \mathrm{p}=0.001$, partial $\eta 2=$ 0.489 ) remained significant, we could not adjust for the effect of confounding variables including psychiatric comorbidities because of small sample size. Fourth, we did not conduct structured interviews for the diagnoses of psychiatric disorders. Fifth, the adverse events might be under-reported, as we relied on the self-reporting of such effects by the subjects or their parents/guardians.

Despite these caveats, our results provide preliminary evidence of the effectiveness and safety of clonidine ER. Large, prospective, placebo-controlled studies are needed to support these findings.

\section{Supplementary Materials}

The online-only Data Supplement is available with this article at https://doi.org/10.4306/pi.2018.15.1.90.

\section{Acknowledgments}

This research was supported by the Basic Science Research Program, through the National Research Foundation of Korea (NRF), funded by the Ministry of Science, ICT \& Future Planning (2015R1A1A3A04001349). 


\section{REFERENCES}

1. Hirota T, Schwartz S, Correll CU. Alpha-2 agonists for attention-deficit/hyperactivity disorder in youth: a systematic review and metaanalysis of monotherapy and add-on trials to stimulant therapy. J Am Acad Child Adolesc Psychiatry 2014;53:153-173.

2. Schwartz S, Correll CU. Efficacy and safety of atomoxetine in children and adolescents with attention-deficit/hyperactivity disorder: results from a comprehensive meta-analysis and metaregression. J Am Acad Child Adolesc Psychiatry 2014;53:174-187.

3. Faraone SV. Using Meta-analysis to compare the efficacy of medications for attention-deficit/hyperactivity disorder in youths. P T 2009; 34:678-694.

4. Ganos C, Martino D. Tics and tourette syndrome. Neurol Clin 2015;33: 115-136.

5. Jiao F, Zhang X, Zhang X, Wang J. Clinical observation on treatment of Tourette syndrome in Chinese children by clonidine adhesive patch.
Eur J Paediatr Neurol 2016;20:80-84

6. Cavanna AE, Selvini C, Termine C, Balottin U, Eddy CM. Tolerability profile of clonidine in the treatment of adults with Tourette syndrome. Clin Neuropharmacol 2012;35:269-272.

7. Jain R, Segal S, Kollins SH, Khayrallah M. Clonidine extended-release tablets for pediatric patients with attention-deficit/hyperactivity disorder. J Am Acad Child Adolesc Psychiatry 2011;50:171-179.

8. Kollins SH, Jain R, Brams M, Segal S, Findling RL, Wigal SB, et al. Clonidine extended-release tablets as add-on therapy to psychostimulants in children and adolescents with ADHD. Pediatrics 2011;127:e1406-e1413.

9. Leckman JF, Hardin MT, Riddle MA, Stevenson J, Ort SI, Cohen DJ. Clonidine treatment of Gilles de la Tourette's syndrome. Arch Gen Psychiatry 1991;48:324-328.

10. Kessler AR. Clonidine treatment increases tics in patients with Tourette syndrome: case report. J Child Neurol 2001;16:380-381. 